\title{
Opting for Local Region in SHOX2 Promoter as a DNA Methylation Biomarker for Lung Cancer Diagnosis
}

\author{
Pham Anh Thuy Duong, Nguyen Thu Trang, \\ Nguyen Thuy Ngan, Vo Thi Thuong Lan* \\ VNU University of Science, 334 Nguyen Trai, Thanh Xuan, Hanoi, Vietnam
}

Received 19 September 2021

Revised 02 November 2021; Accepted 13 November 2021

\begin{abstract}
Epigenetic alterations play a main role in the initiation and progression of lung cancer. CpG methylation in the promoter of the Short Stature Homeobox 2 (SHOX2) gene has been evaluated and validated at different stages of this malignant disease using quantitative methylation-specific PCR (qMSP) method. This is a simple, fast, and cost-effective technique that can be easily applied to clinical practice. In this study, formalin-fixed, paraffin-embedded (FFPE) tissue samples were collected from 30 lung cancer patients and 30 patients suffering from non-cancerous pulmonary diseases. The methylation level of $S H O X 2$ was evaluated in two CpG-riched regions of the promoter by using qMSP. The SHOX2 methylation level of both regions in lung cancer was significantly higher than that in non-cancerous lung diseases $(23.62 \%$ versus $0.23 \%$, and $8.52 \%$ versus $0.65 \%$, respectively), indicating that $\mathrm{SHOX} 2$ methylation could be conferred as a potential biomarker to lung cancer.
\end{abstract}

Keywords: DNA methylation, SHOX2, lung cancer, quantitative methylation-specific PCR (qMSP).

\section{Introduction}

DNA methylation occurring at $\mathrm{CpG}$ dinucleotides that frequently locate in promoter regions is well known as an epigenetic regulation mechanism for transcriptionally silencing gene expression [1]. Hypermethylation usually occurs at the promoter region which can drive the silencing of key tumor suppressors [2, 3]. Aberrant DNA

\footnotetext{
* Corresponding author.

E-mail address: lanvtt@vnu.edu.vn

https://doi.org/10.25073/2588-1140/vnunst.5333
}

methylation is the earliest molecular alteration occurring during carcinogenesis and is specific for the malignant state; therefore, for a long time, it has been considered a powerful potential biomarker for diagnosis, prognosis, and prediction of cancer diseases [4, 5]. Currently, commercially available IVD tests by the type of methylation-based biomarker have been applied for diagnosis, prognosis, and predictive of various types of cancers such as lung, breast, cervical, colorectal, prostate, and even cancers of the unknown primary site [6].

Lung cancer is the leading cause of cancer-related mortality worldwide [7]. The 
most biomarkers widely used for lung cancer diagnosis is serum biomarker and low-dose CT screening, which have the high false-positive rates [8]. Therefore, investigating DNA methylation as a biomarker for lung cancer detection has been extensively investigated. Currently, several valuable DNA methylation markers have been evaluated and validated at different stages of lung cancer and across ethnicities $[9,10]$. At present, the Epi proLung BL Reflex Assay® (Epigenomics AG, Berlin, Germany), a CE-IVD test for quantifying methylation level of the Short Stature Homeobox 2 (SHOX2) using methyl-specific PCR showed a sensitivity $78 \%$ and specificity $96 \%$ in lung cancer detection using tissue specimens [11].

In Vietnam, the burden of cancer has been rising rapidly in recent years. Lung cancer is also the leading cause of cancer-related mortality and can reach double incidence in 2025 [12]. Therefore, investigating and evaluating DNA methylation as a powerfully auxiliary biomarker for cancer in general and lung cancer, in particular, is urgently needed. Preliminary research on qualitative DNA methylation of genes involved in breast, colorectal cancer has been previously described to Vietnamese patients; however quantitative analysis of specific methylation levels through real-time PCR has not been performed yet [13]. The most important ensuring the clinic value of DNA methylation marker is primer sets using for quantitatively specific methylation real-time PCR (qMSP-PCR) reaction must be designed based on $\mathrm{CpG}$ riched sequences whose methylation level should be significantly altered in cancer as comparison with noncancerous or healthy subjects [14].

In this study, using the qMSP-PCR method we investigated the quantitative methylation level at the SHOX2 promoter gene in Vietnamese patients who suffered from cancer and non-cancerous lung diseases. The SHOX2 gene consisted of three promoters and three $\mathrm{CpG}$ islands, one of which overlaps with the first exon and whose methylation level has been extensively investigated in order to developing a biomarker for lung cancer detection $[9,10,15]$. This study aims at opting for $\mathrm{CpG}$ riched sequences in the SHOX2 promoter region and evaluating their methylation levels in these tissues. Furthermore, the comparison of the SHOX2 methylation profiles in lung cancer and non-cancerous lung tissues will highlight the potential value of epigenetic biomarkers to contribute to the effective lung cancer diagnosis in our country.

\section{Methodology}

\subsection{Sample Collection}

Formalin fixed paraffin embedded (FFPE) tissue samples were collected from 30 lung cancer patients and 30 patients suffering from non-cancerous pulmonary diseases (whose classification was examined by pathologists) at the 175 Hospital (Ho Chi Minh City) during 2019 - 2020. Out of 30 lung cancer, 24 were derived from early stage of lung cancer (stage I/II). Informed consent was obtained from healthy participants and patients in written form and the study was approved by the Ethics Committee of Vietnam Academy of Science and Technology (03-2020/NCHG-HDDD).

\subsection{Genomic DNA Isolation and DNA Bisulfite Conversion}

Genomic DNAs were extracted from FFPE lung tissues using the QIAamp DNA FFPE Tissue Kit (Qiagen). Subsequently, genomic DNAs were subjected to bisulfite conversion using the EZ DNA Methylation-Gold kit (Zymo Research). This chemical specifically converts unmethylated cytosine, but not methylated cytosine, to uracil residues [16].

\subsection{Primer Design}

Primer sets for methylation specific PCR method were designed for measuring the methylation level of the $S H O X 2$ promoter region (NG-047079 positions 7750-7730). This 
region overlaps with the first exon and contains the $\mathrm{CpG}$ island whose methylation was altered in lung cancer $[9,10,15]$. Specific primers that are complementary to the sense strand of the bisulfite converted SHOX2 were designed using the Methyl Primer Express Software v1.0. The methylation specific PCR primers used for profiling $S H O X 2$ methylation derived from the CpGs-containing sequence to ensure their specific annealing to the bisulfite treated target. Two reverse primers were derived from two consecutive sequences on the SHOX2 promoter. One forward primer was separately combined with two reverse primers in the qPCR reactions to amplify the methylated SHOX2.1 and SHOX2.2 sequences, respectively. In addition, the classical $\Delta \Delta \mathrm{CT}$ approach using a calibrator reference was used for relative calculation of methylation level; thus, the $\mathrm{CpG}$ free sequence from the actin beta $(A C T B)$ gene was chosen as reference [17]. Primer sequences, amplicon lengths, and qPCR conditions are shown in Table 1.

Table 1. Primer sets and quantitative real time PCR conditions for measurement of SHOX2 methylation

\begin{tabular}{|c|c|c|c|}
\hline Primers & $\begin{array}{l}\text { Sequences } \\
\left(5^{\prime}-3^{\prime}\right)\end{array}$ & $\begin{array}{l}\text { Size } \\
\text { (bp) }\end{array}$ & $\begin{array}{c}\mathrm{qPCR} \\
\text { conditions }\end{array}$ \\
\hline $\begin{array}{l}\text { SHOX- } \\
\text { Me-F }\end{array}$ & $\begin{array}{l}\text { agacgtttttcgttgtt } \\
\text { tttgggttcg }\end{array}$ & \multirow{2}{*}{93} & \multirow{6}{*}{$\begin{array}{l}95^{\circ} \mathrm{C} 5 \\
\mathrm{~min}, 40 \\
\text { cycles of } \\
\left(95^{\circ} \mathrm{C} 10\right. \\
\mathrm{sec}, 63^{\circ} \mathrm{C} \\
30 \mathrm{sec}, \\
72^{\circ} \mathrm{C} 30 \\
\sec ), 72^{\circ} \mathrm{C} \\
5 \mathrm{~min} .\end{array}$} \\
\hline $\begin{array}{l}\text { SHOX- } \\
\text { Me-R1 }\end{array}$ & $\begin{array}{l}\text { acgaccccgatcga } \\
\text { acaaacgaaacg }\end{array}$ & & \\
\hline $\begin{array}{l}\text { SHOX- } \\
\text { Me-F }\end{array}$ & $\begin{array}{l}\text { agacgtttttcgttgtt } \\
\text { tttgggttcg }\end{array}$ & \multirow{2}{*}{102} & \\
\hline $\begin{array}{l}\text { SHOX- } \\
\text { Me-R2 }\end{array}$ & $\begin{array}{l}\text { cgaccaacataacgt } \\
\text { aaacgcctatactcg }\end{array}$ & & \\
\hline ACTB-F & $\begin{array}{l}\text { aggaggtttagtaag } \\
\text { ttttctggattg }\end{array}$ & \multirow{2}{*}{104} & \\
\hline ACTB-R & $\begin{array}{l}\text { cccttaaaaattacaa } \\
\text { aaaccacaaccta }\end{array}$ & & \\
\hline
\end{tabular}

\subsection{Cloning the Bisulfite Converted ACTB and} Methylated SHOX2 Sequences

The bisulfite converted $A C T B$ and methylated $S H O X 2$ sequences were amplified from bisulfite converted DNA extracted from lung cancer sample, purified by GeneJET PCR
Purification Kit (Thermo Scientific) and then cloned using InsTAclone PCR Cloning Kit (Thermo Scientific). The inserts in recombinant plasmids were sequenced (3500 Genetic Analyzer).

\subsection{Quantitative Real Time PCR Assay}

The real-time PCR was carried out in $20 \mu \mathrm{l}$ per reaction using bisulfite converted DNA as template and SsoAdvanced Universal SYBR Green Supermix (Biorad). Real-time PCR assays were duplexed for each sample. Water with no DNA template was included in each PCR reaction as a control for contamination. All qPCR reactions were performed using the 7500 Real-time PCR instrument (Applied Biosystems, CA).

\subsection{Methylation Calculation}

The SHOX2 methylation level was calculated by using the $\Delta \Delta \mathrm{CT}$ method that requires a calibrator sample with a defined methylation level. In this study, the defined methylation level of $10 \%$ was obtained by mixing linearized pACTB and pMe-SHOX plasmids. A serial dilution of the linearized recombinant plasmids pACTB and pMe-SHOX containing bisulfite converted $A C T B$ and methylated SHOX2 sequences, respectively were used for determination of cut-off value for the measurement of SHOX2 methylation level.

\subsection{Statistical Analysis}

Data was processed by using Microsoft Office 365 software (Microsoft), then analyzed by GraphPad Prism 9.0.0 software package (GraphPad Software LLC). In all boxplots, methylated SHOX2 level was expressed as medians with interquartile values. Simple linear regression fits a straight line through $\mathrm{Ct}$ values of a serial concentration of plasmids to find the best-fit value of the slope and intercept. Comparisons between two groups on the methylation level were assessed by using the Mann-Whitney $U$ test and were graphed in the box-and-whisker plot format. A P-value $<0.05$ was considered statistically significant. 


\section{Results and Discussion}

\subsection{Specificity of the Designed Primers}

In order to confirm the accuracy of the methylated specific primer derived from the SHOX2 promoter sequences, the PCR product amplified by the SHOX-Me-F/SHOX-Me-R2 primer pair and successfully cloned into plasmids pMe-SHOX was sequenced. The nucleotide sequence presenting in Figure 1 showed that all cytosines in the $\mathrm{CpG}$ sites remained to be cytosines and the cytosines alone were converted to thymines. This result confirmed that the designed primer sets were specific to the methylated $\mathrm{SHOX2}$ sequence.

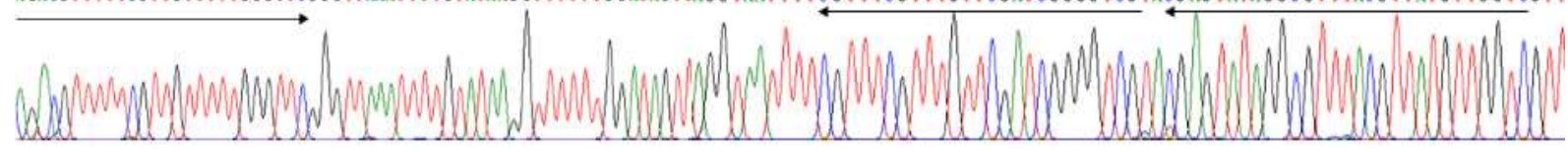

Figure 1. Nucleotide sequence of the insert in pMe-SHOX plasmid. Nucleotides present in the primers were arrowed. All cytosines in the $\mathrm{CpG}$ sites remain cytosines while the cytosines alone were converted to thymines.

\section{2. qPCR Amplication Efficiency}

In order to quantify the amplification efficiency, a serial dilution of two linearized pACTB, pMe-SHOX2 plasmids were used as templates in qPCR reactions. In Figure 2, the CT value is plotted according to the serial plasmid concentrations (from $10^{1}$ to $10^{4}$ copies/reaction), showing high amplification eficiency of qPCR, thus meaning that $\Delta \Delta C T$ calculation was suitable to analyze the methylated SHOX2 level of two SHOX2.1 and SHOX2.2 regions.
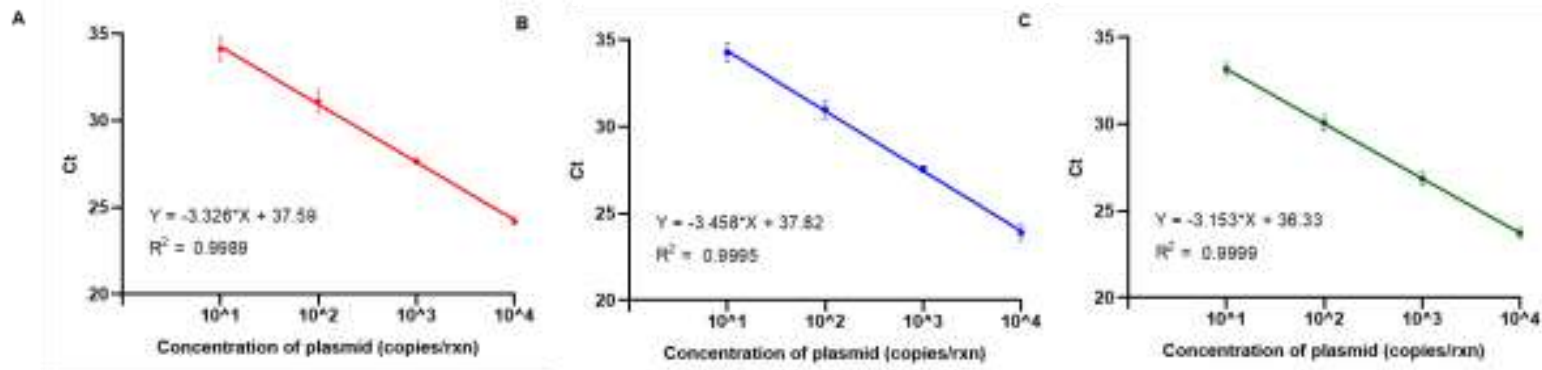

Figure 2. Analytical performance of the SHOX2 qPCR assay. A serial concentrations of the plasmids pACTB and pMe-SHOX were used as templates for qPCR reactions amplified with primer pair ACTB-F/ACTB-R (A), SHOX-Me-F/SHOX-Me-R1 (B) and SHOX-Me-F/SHOX-Me-R2 (C), respectively. The standard curves of these plasmids were built based on the CT values, using simple linear regression analysis. Each point of curves was replicates 4 times and the standard deviation is indicated by error bars.

3.3. Analysis of SHOX2.1 and SHOX2.2 Methylation Levels in Patients with Lung Cancer and Non-cancerous Lung Diseases

In order to investigate the methylation profile of $S H O X 2$, two regions enriched CpGs on the promoter (positions 7750 - 7730, NG-047079), SHOX2.1 and SHOX2.2, was chosen to analysis on 30 FFPE samples (30 patients with lung cancer versus 30 patients with non-cancerous lung diseases). Descriptive statistics of SHOX2.1 and SHOX2.2 methylation were shown in Table 2 . The methylation level of both regions was low in non-cancerous lung diseases $(0.23$ and 0.65$)$ (Figure 3A) but significantly increased in lung cancer, and the methylation level of SHOX2.1 was significantly higher than that of SHOX2.2 (23.62 versus 8.52 , respectively) (Figure $3 \mathrm{~B}$ ). Both regions were dramatically hypermethylated in lung cancer (Figure 3C, D). 
Table 2. Descriptive statistics of $S H O X 2.1$ and SHOX2.2. NC: non-cancerous lung diseases, LC: lung cancer

\begin{tabular}{|l|r|r|r|r|}
\hline \multirow{2}{*}{ Descriptive statistics } & \multicolumn{2}{|c|}{ SHOX2.1 } & \multicolumn{2}{c|}{ SHOX2.2 } \\
\cline { 2 - 5 } & NC & LC & NC & LC \\
\hline $\begin{array}{l}\text { Total number } \\
\text { of values }\end{array}$ & 30 & 30 & 30 & 30 \\
\hline Minimum (\%) & 0.02 & 8.99 & 0.02 & 3.51 \\
\hline 25\% Percentile (\%) & 0.03 & 13.937 & 0.02 & 4.56 \\
\hline Median (\%) & 0.23 & 23.62 & 0.65 & 8.52 \\
\hline $75 \%$ Percentile (\%) & 1.27 & 39.99 & 1.08 & 33.66 \\
\hline Maximum (\%) & 4.77 & 59.95 & 7.17 & 67.68 \\
\hline Mean (\%) & 0.82 & 26.91 & 1.27 & 20.33 \\
\hline Std. Deviation (\%) & 1.20 & 15.30 & 1.89 & 20.39 \\
\hline $\begin{array}{l}\text { Std. Error } \\
\text { of Mean (\%) }\end{array}$ & 0.22 & 2.80 & 0.35 & 3.722 \\
\hline $\begin{array}{l}\text { Lower 95\% CI } \\
\text { of mean (\%) }\end{array}$ & 0.38 & 21.20 & 0.563 & 12.71 \\
\hline $\begin{array}{l}\text { Upper 95\% CI } \\
\text { of mean (\%) }\end{array}$ & 1.27 & 32.62 & 1.975 & 27.93 \\
\hline
\end{tabular}

Different variance in SHOX2.1 and SHOX2.2 methylation could be explained by potential transcription factor binding sites for SHOX2 promoter [15] and epigenetic heterogeneity in cancer [18]. Indeed, interaction of transcription factor $\mathrm{Tbx} 4$ with three binding sites on the SHOX2 promoter in limb development has been described previously [19]. It has been reported that not all CpG sites within a single promoter region are functionally equivalent in transcriptional regulation; thus, the precise location of clinically relevant methylated CpGs plays an important role in the development of a DNA methylation-based biomarker [20]. Moreover, epigenetic variation between cancer cells within a tumor of the same patient (intratumor heterogeneity) is a remarkable tumor stages [21]. Our result was in line with previous reports on SHOX2 hypermethylation in lung cancer. Moreover, SHOX2.1 was higher methylated than SHOX2.2 in lung cancer, thus allowing better discrimination of lung cancer from non-cancerous lung diseases.
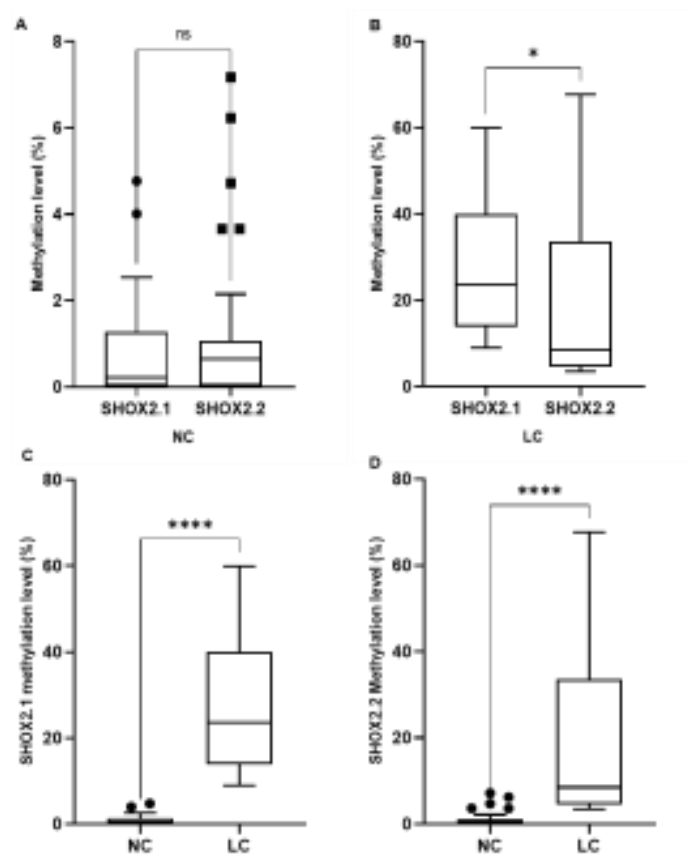

Figure 3. SHOX2 methylation. Methylation level between $S H O X 2.1$ and $S H O X 2.2$ in non-cancerous lung diseases (NC) (A) and in lung cancer (LC) (B). Hypermethylation was observed in $\mathrm{SHOX} 2.1$ (C) and SHOX2.2 (D) in NC and LC. Mann Whitney test was used to compare the difference for two groups (*) $\mathrm{p}<0.05,(* * * *) \mathrm{p}<0.0001, \mathrm{~ns}-$ nonsignificant.

\section{Conclusion}

To summarize, this study has chosen the quantitative qMSP-PCR method for the preliminary analysis of SHOX2 methylation. We have showed that hypermethylation in SHOX2.1 sequence could be conferred as a potential biomarker to lung cancer. These encouraging results prompt us to extend the SHOX2.1 methylation analysis in noninvasive liquid biopsy samples, in the common effort to foster the use of DNA methylation analysis in biomarker development and clinical applications.

\section{Acknowledgements}

The authors would like to thank the Center for Life Science Research (VNU University of Science) for technical support. This 
study was financially supported by Grant 108.01-2019.306 from the Ministry of Science and Technology, Vietnam.

\section{References}

[1] L. E. Blake et al., A Comparison of Gene Expression and DNA Methylation Patterns Across Tissues and Species, Genome Res, Vol. 30, No. 2, 2020, pp. 250-262, https://doi.org/10.1101/gr.254904.119.

[2] S. B. Baylin, DNA Methylation and Gene Silencing in Cancer, Nat Clin Pract Oncol, Vol. 2, No. S1, 2005, pp. 4-11, https://doi.org/10.1038/ncponc0354.

[3] G. Malpeli et al., Methylation Dynamics of RASSF1A and Its Impact on Cancer, Cancers (Basel), Vol. 11, No. 7, 2019, https://doi.org/ 10.3390/cancers11070959.

[4] W. J. Locke et al., DNA Methylation Cancer Biomarkers: Translation to the Clinic, Front Genet, Vol. 10, 2019, pp. 1150, https://doi.org/ 10.3389/fgene.2019.01150.

[5] H. Kim, X. Wang, P. Jin, Developing DNA Methylation-based Diagnostic Biomarkers, J. Genet Genomics, Vol. 45, No. 2, 2018, pp. 87-97, https://doi.org/10.1016/j.jgg.2018.02.003.

[6] O. T. Lesniak, K. E. Sokolowska, T. K. Wojdacz, Current Satus of Development of Methylation Biomarkers for in Vitro Diagnostic IVD Applications, Clin Epigenetics, Vol. 12, No. 1, 2020, pp. 100, https://doi.org/10.1186/s13148020-00886-6.

[7] H. Sung et al., Global Cancer Statistics 2020: GLOBOCAN Estimates of Incidence and Mortality Worldwide for 36 Cancers in 185 Countries, CA Cancer J. Clin, Vol. 71, No. 3, 2021, pp. 209-249, https://doi.org/10.3322/caac.21660.

[8] R. Fang et al., The Evaluation of Serum Biomarkers for Non-small Cell Lung Cancer (NSCLC) Diagnosis, Front Physiol, Vol. 9, 2018, pp. 1710 , https://doi.org/10.3389/fphys.2018.01710.

[9] Y. X. Shi et al., Current Landscape of Epigenetics in Lung Cancer: Focus on the Mechanism and Application, J. Oncol, Vol. 20, 2019, pp. 8107318, https://doi.org/10.1155/2019/8107318.

[10] B. Schmidt et al., SHOX2 DNA Methylation is a Biomarker for the Diagnosis of Lung Cancer Based on Bronchial Aspirates, BMC Cancer, Vol. 10, 2010, pp. 600, https://doi.org/10.1186/1471-2407-10-600.
[11] D. Dietrich et al., Development and Performance Evaluation of a CE-IVD for Measuring SHOX2 DNA Methylation in Bronchial Aspirates for the Diagnosis of Lung Cancer, Lung Cancer, Vol. 77, 2012, pp. 22, https://doi.org/ 10.1016/j.lungcan.2012.05.036.

[12] S. M. Nguyen et al., Projecting Cancer Incidence for 2025 in the 2 Largest Populated Cities in Vietnam, Cancer Control, Vol. 26, No. 1, 2019, pp. 1073274819865274 , https://doi.org/ 10.1177/1073274819865274.

[13] P. K. Truong et al., BRCA1 Promoter Hypermethylation Signature for Early Detection of Breast Cancer in the Vietnamese Population, Asian Pac, J. Cancer Prev, Vol. 15, No. 22, 2014, pp. $9607-9610$, https://doi.org/ 10.7314/apjcp.2014.15.22.9607.

[14] E. Khodadadi et al., Current Advances in DNA Methylation Analysis Methods, Biomed Res Int, Vol. 2021, 2021, pp. 8827516, https://doi.org/10.1155/2021/8827516.

[15] N. Li, Y. Zeng, J. Huang, Signaling Pathways and Clinical Application of RASSF1A and SHOX2 in Lung Cancer, J. Cancer Res Clin Oncol, Vol. 146, No. 6, 2020, pp. 1379-1393, https://doi.org/ 10.1007/s00432-020-03188-9.

[16] S. J. Clark et al., High Sensitivity Mapping of Methylated Cytosines, Nucleic Acids Res, Vol. 22, No. 15, 1994, pp. 2990-2997, https://doi.org/10.1093/nar/22.15.2990.

[17] K. J. Livak, T. D. Schmittgen, Analysis of Relative Gene Expression Data using Real-time Quantitative PCR and the 2(-Delta Delta C(T)) Method, Methods, Vol. 25, No. 4, 2001, pp. 402-408, https://doi.org/10.1006/meth.2001.1262.

[18] M. Guo et al., Epigenetic Heterogeneity in Cancer, Biomark Res, Vol. 7, 2019, pp. 23, https://doi.org/10.1186/s40364-019-0174-y.

[19] A. Glaser et al., Tbx4 Interacts with the Short Stature Homeobox Gene Shox2 in Limb Development, Developmental Dynamics, Vol. 243, 2014, pp. 629, https://doi.org/10.1002/DVDY.24104.

[20] R. Wasserkort et al., Aberrant Septin 9 DNA Methylation in Colorectal Cancer is Restricted to a Single CpG Island, BMC Cancer, Vol. 13, 2013, pp. 398, https://doi.org/10.1186/1471-2407-13-398.

[21] Y. Assenov et al., Intratumor Heterogeneity in Epigenetic Patterns, Seminars in Cancer Biology, Vol. 51, 2018, pp. 12. https://doi.org/10.1016/j.semcancer.2018.01.010. 